\title{
The Neutrophil to Lymphocyte Ratio is an Independent Prognostic Factor in Patients with Metastatic Gastric Cancer
}

\author{
Fatma Yalcın Musri ${ }^{1 *}$, Hasan Mutlu ${ }^{1}$, Melek Karakurt Eryılmaz ${ }^{1}$, Derya Kıvrak \\ Salim $^{1}$, Seyda Gunduz ${ }^{2}$, Hasan Senol Coskun ${ }^{1}$
}

\begin{abstract}
Inflammation can play an important role in cancer progression and the prognostic importance of neutrophil to lymphocyte ratio (NLR), a marker of inflammation, in cancer is a current investigation topic. In the present study, we aimed to determine whether there is a prognostic link between NLR and metastatic gastric cancer (mGC). A total of 143 patients from the Akdeniz University and Antalya Training and Research Hospital database were retrospectively analyzed. The median NLR value was 3.34. The median overall survival (OS) and median progression-free survival (PFS) were 11.6 and 7.9 months, respectively, in patients with NLR $<3.34$ while these values were 8.3 and 6.2 months respectively in patients with $N L R>3.34(p<0.001$ and $p=0.011$, respectively). Our study showed that increased NLR is an independent prognostic factor associated with short survival in patients with mGC.
\end{abstract}

Keywords: Gastric cancer - neutrophil-to-lymphocyte ratio - progression-free survival - overall survival

Asian Pac J Cancer Prev, 17 (3), 1309-1312

\section{Introduction}

Gastric cancer (GC) is the fourth most frequent malignancy and the second leading cause of cancerrelated mortality worldwide (Herszenyi et al, 2010). Patients are generally diagnosed at metastatic stage, and the prognosis at this stage is poor with "very low 5-year survival rates". Nevertheless, the basic treatment is systemic chemotherapy and survival rates vary depending on palliative chemotherapy. Therefore, like other malignancies, determination of prognostic factors is a fairly matter of interest also in patients with metastatic gastric cancer.

Both tumor-related and host-related factors, especially systemic inflammatory response are important in cancer development (Colotta et al., 2009 ; Hanahan et al., 2011). C-reactive protein (CRP), hypoalbuminemia , increased levels of some cytokines, increased leukocytosis and its subtypes in peripheral blood can reflect the systemic inflammatory response ( Vigano et al., 2000; Roxburgh et al., 2010). Peripheral neutrophil to lymphocyte ratio (NLR) is an easily measurable parameter which reflects systemic inflammation. It has been demonstrated that increased NLR is associated with poor prognosis and predicts short survival in various cancer types (Templeton et al., 2014).

In our study, we aimed to demonstrate whether NLR has a prognostic importance in terms of survival in patients with metastatic gastric cancer (mGC).

\section{Materials and Methods}

A total of 143 patients with mGC from Akdeniz University and Antalya Training and Research Hospital database were evaluated retrospectively. The median number of neutrophil to lymphocyte ratio at diagnosis was found as 3.34. The patients were divided into two groups according to median number of NLR at diagnosis: NLR<3.34 (n:72) and NLR>3.34 (n:71).

The age, sex, comorbidity, smoking, alcohol consumption, tumor grade, lymphovascular invasion (LVI), pretreatment albumin and CA 19-9 levels, metastatic area, first-line chemotherapy regimen, secondline chemotherapy (yes or no) were imported into the Statistical Package for the Social Sciences version 16.0 (SPSS 16.0) from the medical archives retrospectively. In addition, the date of diagnosis, date of progression under first-line chemotherapy and date of death of patients with $\mathrm{mGC}$ were imported into the SPSS 16.0 statistical program. Overall survival was defined as the time from the beginning of treatment to death.

Statistical analyses were performed using SPSS software version 16.0. To determine the properties in patients, frequency was performed. Comparisons of patient and tumor characteristics were performed using the chi-square test and the two independent samples $\mathrm{t}$ 


\section{Fatma Yalcin Musri et al}

test. The effect of NLR on OS of patients with mGC was investigated using the log-rank test. Kaplan-Meier survival estimates were calculated. Univariate analysis was performed using Cox proportional hazards regression modeling. The parameters those their $\mathrm{p}$ values $<0.250$ were included in multivariate analysis. A p value of $<0.05$ was considered to be statistically significant.

\section{Results}

The properties of groups are shown in Table 1. The mean age was younger in group with $\mathrm{NLR}<3.34$ than other with NLR>3.34 $(\mathrm{p}=0.025)$. There was not a significant difference between groups regarding sex, comorbidity, alcohol consumption, tumor grade, LVI, albumin, CA 19-9, metastatic area, first-line chemotherapy, and second-line chemotherapy $(\mathrm{p}=0.150, \mathrm{p}=0.106, \mathrm{p}=0.057$, $\mathrm{p}=0.189, \mathrm{p}=0.981, \mathrm{p}=0.121, \mathrm{p}=0.901, \mathrm{p}=0.221, \mathrm{p}=0.352$ and $\mathrm{p}=0.160$, respectively). The ratio of patients smoking in group with NLR>3.34 was significantly higher than other group $(\mathrm{p}=0.046)$.

Table 1. The Properties of Patients According to Median Neutrophil to Lymphocyte Ratio < or > 3.34

\begin{tabular}{|c|c|c|c|}
\hline & $\begin{array}{c}\mathrm{NLR}<3.34 \\
(\mathrm{n}: 72)\end{array}$ & $\begin{array}{c}\text { NLR }>3.34 \\
(n: 71)\end{array}$ & $\mathrm{P}$ \\
\hline Age (mean) & $56.5 \pm 11.8$ & $61.1 \pm 11.8$ & 0.025 \\
\hline Sex & & & 0.150 \\
\hline Female & $33.30 \%$ & $22.50 \%$ & \\
\hline Male & $66.70 \%$ & $77.50 \%$ & \\
\hline Comorbidity & & & 0.106 \\
\hline Yes & $23.20 \%$ & $35.80 \%$ & \\
\hline No & $76.80 \%$ & $64.20 \%$ & \\
\hline Smoking & & & 0.046 \\
\hline Yes & $20.90 \%$ & $40.40 \%$ & \\
\hline No & $79.10 \%$ & $59.60 \%$ & \\
\hline Alcohol Consumption & & & 0.057 \\
\hline Yes & $4.70 \%$ & $17.40 \%$ & \\
\hline No & $95.30 \%$ & $82.60 \%$ & \\
\hline Grade & & & 0.189 \\
\hline 1 & $27.30 \%$ & $41.20 \%$ & \\
\hline 2 & $45.50 \%$ & $17.60 \%$ & \\
\hline 3 & $27.30 \%$ & $41.20 \%$ & \\
\hline LVI & & & 0.981 \\
\hline Positive & $61.10 \%$ & $61.50 \%$ & \\
\hline Negative & $38.90 \%$ & $38.50 \%$ & \\
\hline Pre Albumin (median) & 4 & 3.67 & 0.121 \\
\hline Pre CA 19-9 (median) & 64.1 & 31.5 & 0.901 \\
\hline Metastatic area & & & 0.221 \\
\hline Only Liver & $25.40 \%$ & $32.80 \%$ & \\
\hline Only Lung & $3 \%$ & $1.60 \%$ & \\
\hline Only Peritoneal & $31.30 \%$ & $14.10 \%$ & \\
\hline Only Bone & $3 \%$ & $7.80 \%$ & \\
\hline Other & $14.90 \%$ & $18.80 \%$ & \\
\hline$\geq 2$ organs & $22.40 \%$ & $25 \%$ & \\
\hline First-line CT & & & 0.352 \\
\hline $\mathrm{TCF}$ & $75.40 \%$ & $70.60 \%$ & \\
\hline Platinum+Capecitabine & $1.40 \%$ & $7.40 \%$ & \\
\hline Herceptin based & $8.70 \%$ & $5.90 \%$ & \\
\hline Other & $14.50 \%$ & $16.20 \%$ & \\
\hline Second-line CT & & & 0.160 \\
\hline Yes & $40.80 \%$ & $29.60 \%$ & \\
\hline No & $59.20 \%$ & $70.40 \%$ & \\
\hline
\end{tabular}

The median PFS was significantly higher in the patients with NLR $<3.34$ ( $\mathrm{p}=0.011)$. While the median PFS was 7.9 [95\% CI 5.8-10.0] months in patients with NLR<3.34, it was 6.2 [95\% CI 5.5-6.9] months in patients with NLR $>3.34$. The PFS curves were shown in Figure 1. When we evaluated the groups in terms of OS, the median OS was significantly higher in the patients with NLR $<3.34$ $(\mathrm{p}<0.001)$. While the median OS was 11.6 [95\% CI 8.3514.9] months in patients with NLR $<3.34$, it was 8.3 [95\% CI 5.5-11.0] in patients with NLR $>3.34$. The OS curves were shown in Figure 2.

The age, sex, comorbidity, smoking, alcohol consumption, grade, LVI, pretreatment albumin, pretreatment $\mathrm{Ca}$ 19-9, metastatic area, first-line chemotherapy regimen and second-line chemotherapy, and median NLR ( $<3.34$ or $>3.34$ ) were included univariate analysis and $\mathrm{p}$ values for these factor were given in Table 2. The factors that their $\mathrm{p}$ values were $<0.250$ were included in multivariate analysis. After univariate, comorbidity, alcohol consumption, albumin, CA 19-9,

Table 2. The Results of Univariate Analysis

\begin{tabular}{lc}
\hline & P value \\
\hline Age & 0.809 \\
Sex & 0.593 \\
Comorbidity & 0.231 \\
Smoking & 0.471 \\
Alcohol Comsumption & 0.160 \\
Grade & 0.406 \\
LVI & 0.738 \\
Albumin & 0.234 \\
CA 19-9 & 0.094 \\
Metastatic area & 0.315 \\
First-line CT regimen & 0.483 \\
Second-line CT (yes or no) & $<0.001$ \\
mNLR & $<0.001$ \\
\hline
\end{tabular}

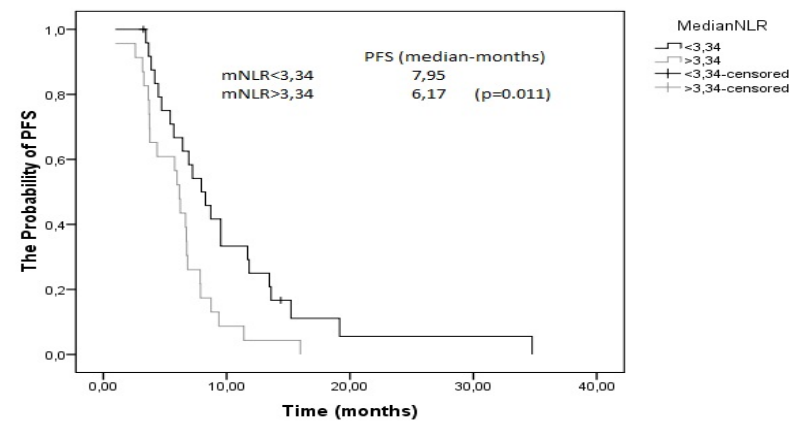

Figure 1. Progression Free Survival (PFS) Curves

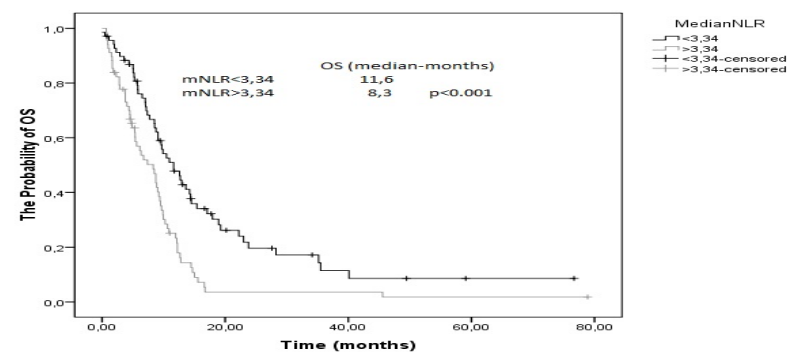

Figure 2. Overall Survival (OS) Curves 
Neutrophil to Lymphocyte Ratio as an Independent Prognostic Factor in Patients with Metastatic Gastric Cancer

second-line chemotherapy and median NLR $(<3.34$ or $>3.34$ ) were included in multivariate analysis. In the final multivariate analysis, median NLR $(<2.93$ or $>2.93)$, CA 19-9 and second-line chemotherapy (yes or no) were found significant ( $\mathrm{p}=0.005, \mathrm{p}=0.029$ and $\mathrm{p}<0.001$, respectively).

\section{Discussion}

In our study, we aimed to investigate the prognostic effect of NLR on PFS and OS in patients with mGC. Our results showed that NLR is an independent prognostic factor on OS $(\mathrm{p}<0.001)$ and PFS $(\mathrm{p}=0.011)$ in patients with mGC.

The link between increased NLR and poor prognosis is based on the difference in neutrophil and lymphocyte functions in the host's immunity. Neutrophils stop the inflammatory response of the immune system through suppression of the cytolytic activity of immune cells such as lymphocytes, activated T-cells and natural killer cells. Neutrophils are also the source of the angiogenetic and growth factors which contribute to tumor progression (Grivennikov et al., 2010). Lymphocytes are the main cells generating the host's immune response against tumor cells, and can attack and destroy cancer cells (Smyth et al., 2006). Patients with lymphocyte-infiltrating tumors have better prognosis than those with non-infiltrating tumors ( Martinet et al., 2011), and lymphopenia is an independent poor prognostic factor for survival in patients with cancer (Fogar et al., 2006 ). However, while absolute neutrophil and lymphocyte counts may be affected by various physiological/pathological factors, NLR remains stable and therefore is superior to the other leukocyte subtypes in evaluation of prognosis.

Several studies demonstrate the prognostic importance of increased levels of NLR in peripheral blood in cancer types such as colorectal cancer (Kishi et al., 2009; Ozdemir et al., 2014) , lung cancer (Sarraf etet al., 2009; Kaya et al., 2013), hepatocellular carcinoma (Gomez etet al., 2008), breast cancer (Nakano etet al., 2014) and many more solid tumors. Some studies which investigated the prognostic link between GC and NLR focused on postoperative survival in operable GC patients rather than on mGC (Shimada et al., 2010; Jung et al., 2011).

In a prospective study conducted in 2007 on 1220 patients with mGC the median NLR was 2.58 . In the univariate analysis, it was an important poor prognostic marker in the group with $\mathrm{N} / \mathrm{L}>2.5$ compared to that with $\mathrm{N} / \mathrm{L}<2.5$ ( $\mathrm{p}=0.019$ ). The median survival was 369 days vs. 239 days, and the 1-year survival rates were $50 \%$ vs. $30 \%$ (in the group with $\mathrm{N} / \mathrm{L}<2.5 v s$. that with $\mathrm{N} / \mathrm{L}>2.5$ ). The multivariate analysis also revealed an important link between NLR and survival ( $\mathrm{p}=0.077$ ) (Yamanaka et al., 2007). In a prospective study conducted in 2010 on 1028 operated patients with all stages of gastric cancer to investigate whether increased pre-operative NLR predicts poor prognosis, NLR was found to be associated with poor prognosis in all stages and well as in 159 stage-IV patients ( cutoff NLR 4.0/ $\mathrm{p}=0.005$ ), and it was also found that NLR increases gradually with increased stage (for stage IV, 3.34+- 2.25) (Shimada et al., 2010). In another study on 268 patients, the median NLR was
3.06 in patients with mGC and statistically significantly prolonged PFS $(p=0.001)$ and OS $(p<0.001)$ were found in the group with low NLR $(\mathrm{NLR} \leq 3.0)$ compared to that with high NLR (NLR > 3.0) (Cho et al., 2014). In our study, the median NLR was 3.34 which is compatible with the median values in the literature. In addition, in the analysis based on the patient characteristics, the smoking rate was statistically significantly higher in the group with NLR >3.34 ( $\mathrm{p}=0.046)$. The univariate analysis showed that the median PFS was 6.2 vs. 7.9 months $(\mathrm{p}=0.011)$ and the median OS was 8.3 vs.11.6 months $(\mathrm{p}<0.001)$, respectively. In the multivariate analysis, we demonstrated the statistically significant prognostic importance of median NLR, pretreatment Ca19.9 and receiving or not receiving second line therapy on survival $(\mathrm{p}=0.005$, $\mathrm{p}=0.029$ and $\mathrm{p}<0.001$, respectively).

The major limitations of our study were the insufficient number of patients and that it was a retrospective study. However, we demonstrated that measurement of NLR using a quick, easy and low-cost test based on peripheral blood count can be used as a clinical biological marker and predict prognosis in patients with mGC (available data in the literature is limited in this field). But our findings will need to be confirmed with larger, prospective, randomized studies.

\section{References}

Cho IR, Park JC, Park CH, et al (2014). Pre-treatment neutrophil to lymphocyte ratio as a prognostic marker to predict chemotherapeutic response and survival outcomes in metastatic advanced gastric cancer. Gastric Cancer, 17, 703-10

Colotta F, Allavena P, Sica A, et al (2009). Cancer-related inflammation, the seventh hallmark of cancer: links to genetic instability. Carcinogenesis, 30, 1073-81.

Fogar P, Sperti C, Basso D, et al (2006). Decreased total lymphocytecounts in pancreatic cancer: an index of adverse outcome. Pancreas, 32, 22-8.

Grivennikov SI, Greten FR, Karin M (2010). Immunity, inflammation, and cancer. Cell, 140, 883-99

Gomez D, Farid S, Malik HZ, et al (2008). Preoperative neutrophil-to-lymphocyte ratio as a prognostic predictor aftercurative resection for hepatocellular carcinoma. World J Surg, 32, 1757-62

Hanahan D, Weinberg RA (2011) .Hallmarks of cancer: the next generation. Cell, 144, 646-74.

Herszényi L, Tulassay Z (2010) .Epidemiology of gastrointestinal and liver tumors. Eur Rev Med Pharmacol Sci, 14, 249-58.

Jung MR, Park YK, Jeong O, et al (2011). Elevated preoperative neutrophil to lymphocyte ratio predicts poor survival following resection in late stage gastric cancer. J Surg Oncol, 104, 504-10.

Kaya V, Yildirim M, Demirpence O, et al (2013). Prognostic significance of basic laboratory methods in non- small-celllung cancer. Asian Pac J Cancer Prev, 14, 5473-6.

Kishi Y, Kopetz S, Chun YS, et al (2009). Blood neutrophil-tolymphocyte ratio predicts survival in patients with colorectal liver metastases treated with systemic chemotherapy. Ann Surg Oncol, 16, 614-22.

Martinet L, Garrido I, Filleron T, et al (2011). Human solid tumors contain high endothelial venules: association with $\mathrm{T}$ - and B-lymphocyte infiltration and favorable prognosis in breast cancer. Cancer Res, 71, 5678-87. 
Nakano K, Hosoda M, Yamamoto M, et al (2014) . Prognostic significance of pre-treatment neutrophil: lymphocyte ratio in Japanese patients with breast cancer. Anticancer Res, 34, 3819-24.

Ozdemir Y, Akin ML, Sucullu I, et al (2014). Pretreatment neutrophil/lymphocyte ratio as a prognostic aid in colorectal cancer. Asian Pac J Cancer Prev, 15, 2647-50.

Roxburgh CS, McMillan DC (2010) . Role of systemic inflammatory response in predicting survival in patients with primary operable cancer. Future Oncol, 6,149-63.

Sarraf KM, Belcher E, Raevsky E ,et al (2009). Neutrophil/ lymphocyte ratio and its association with survival after complete resection in non-small cell lung cancer. J Thorac Cardiovasc Surg, 137, 425-8.

Shimada H, Takiguchi N, Kainuma O, et al (2010). High preoperative neutrophil-lymphocyte ratio predicts poor survival in patients with gastric cancer. Gastric Cancer, 13, 170-6.

Smyth MJ, Dunn GP, Schreiber RD (2006). Cancer immunosurveillance and immunoediting: the roles of immunity in suppressing tumor development and shaping tumor immunogenicity. Adv Immunol, 90, 1-50.

Templeton AJ, McNamara MG, Seruga B, et al (2014). Prognostic role of neutrophil-to-lymphocyte ratio in solid tumors: a systematic review and meta-analysis. $J$ Natl Cancer Inst, 106, 124.

Vigano A, Bruera E, Jhangri GS, et al (2000). Clinical survival predictors in patients with advanced cancer. Arch Intern Med, 160, 861-8.

Yamanaka T, Matsumoto S, Teramukai S, et al (2007). The baseline ratio of neutrophils to lymphocytes is associated with patient prognosis in advanced gastric cancer. Oncol, 73, 215-20. 